\title{
USO PACÍFICO DE LA ANTÁRTICA COMO NORMA DE IUS COGENS
}

REGINA I. DÍAZ \& FERNANDO VILLAMIZAR"

\section{RESUMEN}

El objeto de este estudio es comprobar que el principio de uso pacífico de la Antártica obliga a todos los Estados de la comunidad internacional, aunque no sean parte del Tratado de 1959, por ser la prohibición de la amenaza o el uso de la fuerza una norma de ius cogens. El trabajo primero se refiere al Tratado Antártico; luego se explica por qué la norma de ius cogens que prohíbe la amenaza o el uso de la fuerza obliga a todos los Estados de la comunidad internacional, sean o no parte de los instrumentos internacionales que la codifican; $y$, finalmente, se muestra evidencia de la existencia de una opinio iuris cogentis específica respecto del uso pacífico de la Antártica.

PALABRAS CLAVE: Antártica, Tratado Antártico, uso pacífico, ius cogens, prohibición, amenaza, fuerza.

\section{PEACEFUL USE OF THE ANTARCTICA AS IUS COGENS NORM}

\section{ABSTRACT}

The purpose of this study is to verify that the principle of peaceful use of Antarctica obliges all States of the international community, even though not part of the Treaty of 1959, because the prohibition of threat or use of force is an ius cogens rule. The paper first relates to the Antarctic Treaty; then explains why the ius cogens rule prohibiting threat or use of force is applied to all States of the international community, whether or not part of the international instruments that code it; and finally we shown evidence of the existence of a specific opinio juris cogentis regarding the peaceful use of Antarctica.

KEY WORDS: Antarctica, Antarctic Treaty, peaceful use, ius cogens, threat, force.

Investigadora y docente, Universidad Bernardo O’Higgins, Observatorio Regional de Paz y Seguridad. Juan Francisco González 834 Ñuñoa, Santiago de Chile.ridiaz@uc.cl.

* Investigador, Universidad Bernardo O`Higgins, Observatorio Regional de Paz y Seguridad. 178 Rue de l`Universite, Paris 75007, Francia. fvillamizar@ubo.cl. 


\section{INTRODUCCIÓN}

El Tratado Antártico (TA) se firmó en Washington el $1^{\circ}$ de diciembre de 1959 y entró en vigencia el 23 de junio de 1961. Los 12 Estados firmantes fueron: Argentina, Australia, Bélgica, Chile, Francia, Japón, Nueva Zelandia, Noruega, Unión del África del Sur, la otrora Unión de Repúblicas Socialistas Soviéticas, Reino Unido e Irlanda del Norte y Estados Unidos de América. 38 Estados se han adherido con posterioridad. El principal propósito de este Tratado de acuerdo con su Preámbulo es asegurar en interés de toda la humanidad que la Antártica continúe utilizándose siempre exclusivamente para fines pacíficos y que no llegue a ser escenario $u$ objeto de discordia internacional, pues dada la importancia estratégica, climática y geofísica de la región, es de interés general su preservación. Este cometido es claro en el texto del Tratado, de ello se da cuenta en este artículo a través de la revisión del origen histórico de este instrumento internacional $y$ de la manera en que se recoge y queda plasmado el principio de uso pacífico del territorio antártico. Sobre esto no hay duda, sin embargo, la interrogante que surge es si ello obliga sólo a los Estados que son parte en el Tratado o si producto del interés general comprometido el principio vincula a todos los Estados de la comunidad internacional.

En nuestra opinión, todos los Estados del orbe están conminados a hacer un uso pacífico de la Antártica, sean o no parte del TA, pues se trataría de una especificación a un ámbito de aplicación territorial determinado de un principio de ius cogens de carácter general aplicable en todo tiempo y lugar, bajo cualquier circunstancia, cual es la prohibición del uso o amenaza de la fuerza. En otras palabras, esta norma de ius cogens prohibitiva que conlleva una obligación de no hacer en términos generales, puede ser traducida en este ámbito específico en una obligación de hacer consistente en usar pacíficamente el territorio antártico.

En el trabajo se da cuenta del reconocimiento en doctrina y jurisprudencia internacionales del carácter de ius cogens de la norma que prohíbe la amenaza o el uso de la fuerza para luego explicar por qué las normas de esta categoría obligan a todos los Estados de la comunidad internacional, sean o no parte de los instrumentos internacionales que las codifican, enfatizándose que sus características de generalidad y universalidad son las que la dotan de aplicabilidad en todo ámbito aún sin o contra el consentimiento de los Estados.

Finalmente, para demostrar la existencia de una opinio iuris cogentis específica respecto del uso pacífico de la Antártica se alude a las múltiples resoluciones habidas en el seno de la Asamblea General de las Naciones Unidas sobre la Cuestión Antártica, las cuales reafirman el principio del uso pacífico del continente.

\section{EL TRATADO ANTÁRTICO: ORIGEN, HISTORIA Y PRINCIPIOS}

La Antártica, ese continente inhóspito y complejo ha sido objeto de múltiples disputas por su conquista especialmente en la primera mitad del siglo XX. Tras célebres excursiones a ese territorio, como la efectuada entre 1907 y 1909 por Ernest Shackleton y las épicas rivalidades en el verano austral de 1911-1912 entre Robert Falcon Scott y Roald Amudsen, la competencia por el dominio territorial del continente blanco fue en aumento y esto se evidenció en respectivas reclamaciones territoriales, que cronológicamente empezó Gran Bretaña en 1908, y le siguieron Nueva Zelandia (1923), Francia (1924), Australia (1933), Noruega (1939), Chile (1940) y Argentina (1942). De todo el continente blanco, sólo el $15 \%$ no fue reclamado (Cfr. Haddelsey 2012, Blackhall 2012, Scott 2003:473).

Si bien la historia sobre las reclamaciones territoriales en el territorio antártico tuvo una larga discusión a fines del siglo XIX y la primera mitad del siglo XX, el tema se torna más difícil al finalizar la Segunda Guerra Mundial, pues la competencia por la Antártica llegó a niveles considerables de agresión, de manera que la prensa internacional de la época denominó estos eventos como la "disputa por la Antártica" (The Scramble for Antarctica) (Vid. Polar Record 1948:228-240) y algunos diplomáticos denominaron estos eventos como "el problema antártico" (The Antarctic Problem), en el cual la tensión entre Chile, Argentina y el Reino Unido tuvo una preponderancia particular, pues tras las expediciones navales chilenas y argentinas realizadas entre 1946 y 1948 a territorios reclamados por el 
Reino Unido (denominados por los británicos como Falkland Islands Dependencies). Este país protestó e inició una disputa diplomática-judicial ante la Corte Internacional de Justicia (CIJ), que Chile y Argentina revirtieron al desestimar la jurisdicción de dicho tribunal (Ver Hunter 1951; Hayton 1956:590-592; Dodds 2008:50-51).

Ante esta tensión, el Departamento de Estado norteamericano empezó a barajar opciones que evitaran el agravamiento de la tensión (Department of State 1947). La primera opción para las autoridades norteamericanas fue proponer un fidecomiso para la Antártica por parte de la Organización de Naciones Unidas de conformidad con los artículos 75,76, 77 y 79 (Capítulo XII) de la Carta de las Naciones Unidas (1945) (CONU) (Department of State 1948a). El gran inconveniente de esta propuesta era netamente jurídico, particularmente en lo referido al alcance legal de lo establecido por el artículo 76, pues según este precepto el fidecomiso debe fomentar la paz y seguridad internacionales, y también la promoción del desarrollo político, económico, social y educativo de la población que habita los territorios fideicometidos. Como es ampliamente sabido, dadas las características propias del inhóspito territorio antártico, en dicho continente no hay población y por lo tanto no podría establecerse un fideicomiso porque no se cumpliría el objetivo para el cual se instaura esta figura (Department of State 1948b, Samuels 2008:732 y ss.).

Ante los inconvenientes prácticos para aplicar la institución del fidecomiso en el territorio antártico, el Departamento de Estado norteamericano planteó un concepto alternativo, según el cual la Antártica quedaría bajo la autoridad y control directa de los Estados interesados (Department of State 1948b). Es decir, la idea de esta propuesta era generar un condominium independiente de la Organización de Naciones Unidas, pero en colaboración con ésta, cuyo funcionamiento debería ser negociado entre los Estados reclamantes de territorios y Estados Unidos. Se excluiría de plano a la otrora Unión Soviética de esta propuesta (Blum 1994:2).

A esta propuesta se le añadirían dos elementos relevantes, que una vez configurado el TA serán los dos principios rectores de éste y del sistema que en virtud de él se establece. Dichos elementos son: (i) por una parte, la desmilitarización del territorio antártico, lo que constituye el origen del principio de uso pacífico de la Antártica, y

(ii) por otra, la libertad de exploración $e$ investigación científica, que, como veremos más adelante, es el mecanismo que permite acercar posiciones ante las difíciles negociaciones de la Conferencia de Washington de 1959 en la cual se negoció el TA (Department of State 1948c). A fin de poner en ejecución este elemento científico, el Departamento de Estado norteamericano le solicitó a la Academia Nacional de Ciencias de Estados Unidos un informe sobre la viabilidad de realización de programas de investigación científica a gran escala en la Antártica (United States National Academy of Sciences 1949).

Las propuestas de fideicomiso y de condominium fueron discutidas en privado entre Estados Unidos y el Reino Unido. Los británicos tenían reparos en cuanto a la fórmula a emplear para solucionar el tema antártico, es decir en cuanto a la aplicación del fideicomiso o del condominium, y en cuanto al ámbito de extensión territorial, pues la propuesta norteamericana apuntaba a que cualquier acuerdo considerara todo el territorio al sur de los $60^{\circ}$ de Latitud Sur (como finalmente se acogió en el TA), tema que no era del agrado de Londres.

Además, los británicos sostenían que se debían restringir las negociaciones a cuatro Estados: Estados Unidos, Reino Unido, Chile y Argentina, estos dos últimos Estados porque la reclamación territorial británica chocaba físicamente con las reclamaciones chilenas y argentinas. Estados Unidos por su parte pretendía que los acuerdos, cualquiera fuera la fórmula que se empleara, debían incluir a todos los Estados que estuvieran interesados, excepto la Unión Soviética, no sólo a los cuatro propuestos por el Reino Unido (Department of State 1948d).

Esta posición norteamericana de excluir a la otrora Unión Soviética era plenamente comprensible si se analiza el contexto histórico en el que se estaba realizando el diálogo con los británicos. La Unión Soviética no había realizado 
ninguna reclamación territorial y además, basta recordar que en el primer semestre de 1948 la relación entre Estados Unidos y la Unión Soviética estaba en un nivel muy tenso, que se agravó a finales de junio de ese año con el bloqueo soviético a Berlin Occidental. Ante esta circunstancia, es muy coherente que los norteamericanos no quisieran negociar con los soviéticos, no solo sobre el tema antártico, sino que respecto de cualquier otro.

El liderazgo de las negociaciones antárticas durante 1948 siguió a cargo de los Estados Unidos con un diálogo bilateral con el Reino Unido, y con acercamientos bastante profundos con Argentina y Chile. También Estados Unidos inició conversaciones con Australia, Nueva Zelanda, Noruega y Francia y los esfuerzos norteamericanos se inclinaban a convencer a dichos Estados sobre la propuesta del fideicomiso (Department of State 1948e).

El Reino Unido se encaminaba a aceptar la creación de un condominio, mientras que Argentina descartaba de plano las propuestas norteamericanas. Por su parte Chile presentó una contrapropuesta denominada en español Plan Escudero y en inglés Escudero Declaration, en virtud de la cual se creaba un modus vivendi en el que durante cinco años se congelaban todas las reclamaciones territoriales y se potenciaba la cooperación científica (Department of State 1948f). Esta contrapropuesta chilena no tuvo apoyo de los Estados interesados en la cuestión antártica y se desechó en un principio (Barros 1990:855), aunque después fue retomada y perfeccionada para constituirse en el artículo 4 del TA, como se verá más adelante, que es el pilar fundamental del Sistema Antártico.

Ante esta variedad de posiciones, Estados Unidos retoma el tema durante el segundo semestre de 1948 y plantea, por una parte, la promoción de la investigación científica en la Antártica, $y$, por otra parte, un acuerdo encaminado a algún tipo de internacionalización del territorio antártico (Department of State 1948g). Este pronunciamiento fue replicado por varios países, pero los aspectos más relevantes se encuentran en las presentaciones chilena y británica.

1 La cual consideraba "imperialista" la tesis de la posesión efectiva, sin embargo, su posición era contraria a sus pretensiones territoriales en el ártico, en donde
Chile planteó que la propuesta norteamericana sobre la internacionalización de la Antártica no estaba en armonía con el Tratado Interamericano de Asistencia Recíproca (TIAR) suscrito en Río de Janeiro, Brasil, en 1947, y por lo tanto no podía acoger esa propuesta. No obstante esto, para Chile era importante e interesante el aspecto del estímulo internacional a la cooperación científica y la posibilidad de hacer un acuerdo para el intercambio de información científica (Department of State, 1948h). Por su parte el Reino Unido aceptó la propuesta norteamericana como una base para iniciar la discusión y en ese sentido empezó a dialogar con Australia y Nueva Zelandia (Vid. Polar Record 1949:361).

Frente a esta situación, Estados Unidos empezó en marzo de 1949 a considerar el Plan Escudero como forma para solucionar la problemática antártica (Department of State 1949), pero paralelamente dos presiones adicionales que le impidieron avanzar para consensuar una propuesta que solucionara la problemática: por una parte hacer su propia reclamación territorial basado en los aportes en materia de descubrimientos y exploración de la Antártica y de otro lado la latencia de una reclamación territorial de la otrora Unión Soviética ${ }^{1}$, basada en los descubrimientos hechos por la Expedición Imperial rusa realizada entre 1819 y 1820 bajo el mando de von Bellingshausen (Department of State 1948i. Para profundizar sobre la historia soviética en la Antártica. Vid. Boczek 1984). En ese escenario, el Departamento de Estado formula una propuesta propia, basada en el Plan Escudero, pero reflejando sus propios intereses. Esta propuesta es el punto de partida de lo que diez años después será el TA.

La mencionada propuesta se conoció como el Proyecto de Declaración Norteamericana sobre la Antártica (Department of State 1950a), y tendría una duración de cinco a diez años. El Proyecto consideraba: (i) la suspensión de las reclamaciones de soberanía, que era tomado del Plan Escudero; (ii) la cooperación, como mecanismo para beneficiar a los participantes; (iii) el ámbito de aplicación de la propuesta era el territorio al sur de los $60^{\circ}$ de latitud sur, (iv) libertad de investigación

sustentaba sus reclamaciones en virtud del control efectivo que realizaba y rechazaba reclamaciones por descubrimiento. 
científica e intercambio de información, (v) la creación de un comité de carácter administrativo y sin poder de decisión al cual los Estados parte debían rendir informes, (vi) recomendaciones para el tratamiento de los terceros Estados que quisieran hacer investigación científica (Department of State 1950a:905-906).

Concomitante con la propuesta norteamericana, la Unión Soviética envía, el 8 de junio de 1950, un memorando a los Estados Unidos, el Reino Unido, Francia, Noruega, Nueva Zelanda, Australia y Argentina ${ }^{2}$, en el cual proclama que el Gobierno Soviético no reconocería como legal ninguna decisión concerniente al régimen antártico si no se incluía su participación (Department of State, 1950b:913).

Esta proclama soviética debió causar un fuerte tensión a todos los interesados en la cuestión antártica durante gran parte de la década de los cincuenta, porque era una muestra más de la ambición global soviética, que ya se había hecho presente en Berlín en 1948, y que agravaba las relaciones antárticas, posibilitando un conflicto a escala mundial. Además, días después, concretamente el 25 de junio de 1950 a las cuatro de la mañana, la actual Corea del Norte lanzó un ataque de gran envergadura sobre lo que hoy es Corea del Sur, hecho que marcó un hito en la historia de la Guerra Fría, como consecuencia del apoyo soviético a esa intervención y a la respuesta norteamericana.

En 1951 el Departamento de Estado norteamericano envía un proyecto titulado Modus Vivendi a la embajada chilena en Washington (Department of State 1951), basado en el Plan Escudero, que Chile contesta en 1953, pero no hubo una decisión al respecto por dos factores: el primero porque Chile, Argentina y Gran Bretaña iniciaron una competencia para sustentar sus respectivas reclamaciones que generó fricciones de consideración, como por ejemplo los disparos realizados en 1952 por marineros argentinos a científicos británicos que intentaban construir una base en Bahía Esperanza (Howkins 2009:17) y la réplica británica de 1953 con el desmantelamiento

2 Chile no fue incluido porque no tenía relaciones diplomáticas con la Unión Soviética.

3 Para ese entonces se habían llevado a cabo dos versiones, la primera entre 1882 y 1883; y la segunda entre 1932 y 1933. de las bases argentinas y chilenas en la Isla Decepción por parte de los tripulantes del barco británico HMS Snipe mediante el bombardeo de dichas bases (Villamizar 2012:264), pese a que había desde 1949 un acuerdo entre Chile, Argentina y el Reino Unido en el sentido de no enviar barcos de guerra al sur de los $60^{\circ}$ de latitud sur, salvo para los movimientos habituales encaminados a atender los relevos de las bases (Pinochet De la Barra 1981:382).

El segundo factor por el cual la propuesta de modus vivendi queda en incertidumbre, concierne al inicio de los planes de coordinación para llevar a cabo la tercera versión ${ }^{3}$ del Año Geofísico Internacional, que tendría lugar entre julio de 1957 y diciembre de 1958. En efecto, durante los primeros años de la década de los cincuenta las grandes potencias mundiales estaban perfilando todo lo necesario para que el Año Geofísico Internacional fuera una realidad. Después de los pasos iniciales que tuvieron lugar entre 1951 y 1953, la Unión Internacional de Geodesia y Geofísica se reunió en Roma en 1954 para crear un Comité Especial, que sesionaría en París entre el 6 y el 10 de julio de 1955. En dicho Comité Especial se acordó un plan para el establecimiento de bases de investigación antárticas, se buscó asegurar el principio de libertad científica y se aceptó la necesidad de buscar una solución temporal a las disputas territoriales a fin de garantizar la colaboración científica internacional (Dodds 2009:40).

Paralelamente, en 1955 la tensión sobre la cuestión antártica volvió a subir como consecuencia de la acción judicial internacional por parte del Reino Unido en contra de Argentina y de Chile ante la CIJ. Aunque el caso fue removido del listado de la CIJ en marzo de 1956 porque los países latinoamericanos no aceptaron la competencia de esa Corte 4 , la pugna era evidente y las consecuencias impensadas.

Con esa tensión latente Nueva Zelanda formula una propuesta que consistía en dejar la Antártica bajo fideicomiso de las Naciones Unidas (como había sido propuesto anteriormente por los Estados Unidos) y abandonar las reclamaciones

4 Antarctica Case, United Kingdom v. Argentina, y United Kingdom v. Chile International Court of Justice Report of Judgment, Advisory Opinions and Orders 1956, Order of March 16th 1956, p. 8. 
territoriales, pero esa propuesta no tuvo resonancia y quedó como una propuesta más sobre la cuestión antártica (Jacobson 2011:5). Sin embargo, inspirada en la propuesta neozelandesa, la India en la Asamblea General de las Naciones Unidas en $1956^{5}$, plantea un principio que después recogería el TA en su artículo I, que se refiere al uso pacífico de la Antártica, y lo hace en los siguientes términos:

La Antártica, región que abarca más de seis millones de millas cuadradas de territorio, tiene una considerable importancia estratégica, climática y geofísica para el mundo en su conjunto. Con el desarrollo de vías de comunicación más rápidas, el área pronto podría tener una importancia clave en el bienestar $y$ progreso de las naciones. La riqueza mineral de su masa territorial se cree es considerable y sus zonas costeras contienen importantes fuentes alimenticias [...] El Gobierno de la India considera que para poder fortalecer la paz universal, sería apropiado y oportuno para todas las naciones acordar y afirmar que el área será utilizada en su totalidad con fines pacíficos y para el bienestar general (Dodds 1997:138).

Para 1957, Estados Unidos revive la propuesta del condominium, pero le sale al paso una propuesta británica que había sido trabajada con Australia y Nueva Zelanda, y que no difería sustancialmente en los elementos de la fórmula del condominum, pero sí en cuanto a los actores que deberían ser parte de esa o cualquier fórmula que se empleara, pues la propuesta británica incluía a la otrora Unión Soviética como parte integrante de cualquier arreglo (Hayton 1960:352), aspecto que los norteamericanos no contemplaban dada la rivalidad que se vivía en ese momento.

En ese contexto se inicia el Año Geofísico Internacional, que es un evento fundamental para la formación del TA, pese a que muchos le restan importancia (Becker 2010:3; Dodds 2006:60; Scott 2011:52), pues es el escenario en que Estados Unidos y la Unión Soviética tienen

5 No sobra recordar que ese año fue particularmente convulsionado en la historia mundial con dos eventos que pusieron en riesgo la paz mundial, por una parte la Crisis del Suez y por otra parte la Revolución Húngara. un diálogo político ${ }^{6}$ a partir de los postulados de cooperación científica (Berguño \& Elzinga 2010:259 y ss.), de manera que las futuras propuestas norteamericanas en adelante debieron incorporar a la Unión Soviética, especialmente al finalizar el Año Geofísico Internacional, porque el fin del evento supuso una disyuntiva para Estados Unidos: o exigía a la Unión Soviética el cumplimiento de los compromisos acordados en el marco del Año Geofísico Internacional, especialmente el relativo a que las bases tuvieran fines exclusivamente científicos y no militares, o confrontar a la Unión Soviética por el control de la región (Agüero 2010:91), posición que daría lugar a una confrontación bélica de proporciones bíblicas y que evidentemente no era viable en la práctica.

Mientras la ejecución del Año Geofísico Internacional ayudaba a destrabar los conflictos diplomáticos en el territorio antártico y habilitaba el camino a una negociación, los Estados Unidos, a través del Consejo de Seguridad Nacional, fijaban de manera paralela, en 1958, cuatro objetivos de interés nacional sobre la Antártica (NSC 5804/1), que constituían la política norteamericana sobre el continente blanco y que eran:

1. Prevenir el uso de la Antártica para propósitos militares.

2. Proveer libertad de investigación científica.

3. Establecer una administración conjunta ordenada de la Antártica por parte de los Estados directamente involucrados.

4. Preservar la Antártica para fines pacíficos únicamente.

Es así entonces como, con el impulso brindado por el éxito del Año Geofísico Internacional y el establecimiento de la política norteamericana sobre la Antártica, Estados Unidos propone la conferencia diplomática que llevaría, no sin dificultades, a que el 1 de diciembre de 1959 se suscribiera el TA, cuyos pilares son los objetivos norteamericanos comentados y la propuesta del Plan Escudero, que es el actual artículo IV del Tratado (Villamizar 2012:265).

6 El Año Geofísico Internacional fue diseñado como un programa de dieciocho meses que excluía actividades políticas, sin embargo esto no fue cumplido a cabalidad. 
El TA contiene varios principios que son el resultado de todo el proceso diplomático llevado a cabo para su concreción y de los acuerdos necesarios para que la gestión del continente blanco sea viable. Dichos principios son:

1. Uso pacífico del territorio antártico, contenido en el artículo I del TA.

2. Libertad de investigación, contenido en el artículo II del TA.

3. Cooperación Científica Internacional e intercambio de: (i) información sobre los proyectos de programas científicos en la Antártica; (ii) intercambio de personal científico entre las expediciones y estaciones en la Antártica; y (iii) intercambio de observaciones y resultados científicos sobre la Antártica, los cuales estarán disponibles libremente (artículo III del TA).

4. Suspensión de las reclamaciones territoriales, prohibición de realizar nuevas reclamaciones o ampliar las realizadas mientras el TA esté en vigor y ausencia de soberanía en las actividades realizadas en la Antártica (artículo IV TA).

Como se puede visualizar, estos principios tienen un objetivo preciso, que es asegurar que la Antártica sea utilizada de manera pacífica, y se evite así cualquier tipo de conflagración bélica, que además por la cantidad de actores involucrados muy seguramente llevaría a una guerra mundial de dimensiones impensadas. Es por estas razones y por el desarrollo histórico-político revisado, que consideramos que el uso pacífico del territorio antártico tiene un lugar especial en el Derecho Internacional (DI), y que ese lugar especial es el de una norma de ius cogens, tal y como se verá en las secciones posteriores.

Se previene que existe otro principio que no está expresamente declarado en el texto del TA, pero que ha sido fundamental para la sostenibilidad y desarrollo del Sistema Antártico. Ese principio es el del consenso para la toma de decisiones, que se manifiesta concretamente en las Reuniones Consultivas (artículo IX TA). Empero, hay muestras de ese principio en algunas partes del TA, como

7 Se previene que se optó por no dar ejemplos en el articulado de la CVDT, por estimarse que podría llevar en lo establecido en su artículo XII respecto de la modificación del propio tratado: El presente Tratado podrá ser modificado o enmendado, en cualquier momento, con el consentimiento unánime de las Partes Contratantes [...] .

\section{RECONOCIMIENTO INTERNACIONAL DEL CARÁCTER DE IUS COGENS DE LA PROHIBICIÓN DE LA AMENAZA O USO DE LA FUERZA}

El ius cogens internacional es una institución jurídica que aparece en el pensamiento de los internacionalistas en la primera mitad del siglo XX para referir al impedimento de pactar en contrario a los principios internacionales básicos, codificándose esta idea en el artículo 53 de la Convención de Viena sobre el Derecho de los Tratados (1969) (CVDT), como una causal de nulidad de los tratados, aunque se ha de prevenir que encuentra su antecedente en el ius naturale necessarium, noción presente en la obra de los teólogos españoles del siglo XVI reconocidos como los fundadores del DI moderno, Francisco de Vitoria y Francisco Suárez, y en los clásicos Groccio, Wolff y Vattel (Alexidze 1981:228, Gómez 2003:8-13).

La prohibición del uso o amenaza de la fuerza ha sido reconocida como norma de ius cogens. Se observa que durante el trabajo de preparación de la CVDT por parte de la Comisión de Derecho Internacional (CDI) se indica como uno de los ejemplos patentes de este tipo de normas (United Nations 1966:199)7, aunque no siempre fue así concebido, pues en épocas antiguas la fuerza armada se entendía lícita al ser considerada un recurso de última instancia que los Estados podían utilizar a su discreción como medio de solucionar controversias o para alcanzar otros fines en apoyo de su diplomacia. Así la Conferencia de la Paz de la Haya de 1907 estuvo basada en un pleno reconocimiento del ius ad bellum. Esta concepción cambió tras un largo proceso que comenzó después la Primera Guerra Mundial, siendo su antecedente inmediato el Pacto Briand-Kellogg de 1928 y su más evidente codificación de carácter general la CONU de 1945 (Jiménez de Aréchaga 1980:108109).

a una idea errónea respecto del contenido del ius cogens. 
En la jurisprudencia internacional también es posible encontrar evidencia del reconocimiento de la prohibición del uso o amenaza de la fuerza como una norma de ius cogens. Por ejemplo, la CIJ a propósito del caso de las Actividades militares y paramilitares llevadas a cabo por Estados Unidos en Nicaragua (1986) indica que tanto Nicaragua como Estados Unidos, de una manera u otra, reconocían en el principio de la prohibición del empleo de la fuerza, una regla de derecho internacional universalmente reconocida y un principio de ius cogens (CIJ, 1986:190). Luego, en la opinión consultiva sobre la conformidad con el derecho internacional de la declaración unilateral de independencia relativa a Kosovo (2010), observa que la ilegalidad de las declaraciones de independencia de Rhodesia del Sur, Chipre Septentrional, y la República Srpska, no se derivaba de su carácter unilateral, sino del hecho de que iban acompañadas de un uso ilícito de la fuerza o de otras infracciones graves de las normas del derecho internacional general, en particular las de carácter imperativo (ius cogens) (CIJ, 2010:81).

\section{FUERZA OBLIGATORIA DE LAS NORMAS DE IUS COGENS}

Las normas de ius cogens que prohíben el uso o amenaza de la fuerza imperan en todo tiempo y lugar, incluida la Antártica, independiente del consentimiento que hayan prestado o no los Estados a tratados que incluyan el principio, tal como en el caso del TA. Las normas de ius cogens tienen como principales características su imperatividad, su generalidad y su universalidad; estas dos últimas son las que permiten explicar el por qué son vinculantes para todos los Estados de la comunidad internacional sin excepción.

\subsection{La generalidad de las normas de ius cogens}

Esta característica de generalidad se deriva de la pertenencia del ius cogens a la esfera del derecho internacional general. En DI se acostumbra a distinguir entre derecho internacional general $o$ común $y$ derecho internacional particular dependiendo de las personas o sujetos de derecho a quienes se imponen las obligaciones y se confieren

8 Al respecto, es pertinente recordar el artículo 43 de la CVDT, en cuanto la pérdida de vigor de un tratado no los derechos, así el primero comprende normas que son válidas para todos los Estados de la comunidad internacional, mientras que el segundo comprende normas de derecho internacional válidas sólo para ciertos Estados, generalmente aquéllos que han participado directamente de su formación (Virally 1966:15, Betanzos 2009:113).

El derecho internacional general o común se crea de forma consuetudinaria, pero no necesariamente exige una práctica largamente establecida por todos los Estados, ni consentimiento, expreso o implícito, de cada Estado en particular, basta un consenso general, pues se ha de considerar al sujeto de DI "Estado" no de forma individual, sino en su calidad genérica, es decir, no se ha de fijar la atención en cada uno de los Estados considerados aisladamente, sino en la comunidad de Estados; no se trata del actuar un Estado concreto, sino del actuar imputable al género al cual él pertenece (Jiménez de Aréchaga 1980:37).

Las normas de derecho internacional general son bastante limitadas en número y con exigua determinación de su contenido, pues deben adaptarse a grupos sociales constituidos en condiciones de vida y de civilización muy diferentes. El contenido de estas normas se relaciona con una cuestión de interés general para la comunidad internacional, por ello crean obligaciones para todos los Estados, aun respecto de aquellos que no han concurrido expresamente a su formación o que ni siquiera existían en ese momento (Verdross 1966:58, Puceiro 2005:370).

También es pertinente destacar que las normas del derecho internacional particular pueden confirmar o complementar derechos y obligaciones del derecho internacional general, por lo que pueden ser aplicados simultáneamente sin surgir conflicto entre sus normas. Por tanto, los Estados al concluir el TA (derecho internacional particular) simplemente confirmaron o codificaron el principio del uso de la fuerza a un ámbito espacial determinado (el territorio antártico) principio de origen consuetudinario y perteneciente al derecho internacional general, lo importante es considerar que en estas circunstancias (tratado codificando costumbre), la norma consuetudinaria bajo el tratado continúa existiendo y tiene autonomía respecto de las normas del

exime a las partes del cumplimiento de iguales obligaciones que provengan de otra fuente de Derecho Internacional. 
tratado, incluso entre quienes no son partes del mismo o si la base convencional despareciera, por ejemplo por denuncia del tratado ${ }^{8}$ (CIJ 1986:176179).

En consecuencia, cabe indagar acerca de la utilidad del TA en cuanto contempla expresamente como su propósito primordial el uso pacífico de la Antártica, pues aunque el Tratado no existiera o los Estados que actualmente son parte lo denunciaran, igualmente seguiría siendo válida y obligatoria la norma de ius cogens que prohíbe el uso o amenaza de la fuerza en todo tiempo y lugar respecto de todos los Estados de la comunidad internacional. El aporte consiste, del mismo modo que en cualquier codificación, en complementar la norma consuetudinaria, haciéndola más rica en contenido y precisión de su alcance (Carter et al. 2003:128). En específico el TA, señala cuáles actos quedan incluidos en la proscripción del uso de la fuerza: a) toda medida de carácter militar, tal como el establecimiento de bases y fortificaciones militares, la realización de maniobras militares, así como los ensayos de toda clase de armas (artículo $1.1 \mathrm{del}$ Tratado); b) toda explosión nuclear y la eliminación de desechos radiactivos (artículo 5.1 del Tratado); y cuales permitidos: el empleo de personal o equipo militar para investigaciones científicas o para cualquier otro fin pacífico (artículo 1.2 del Tratado). También el tratado crea mecanismos de protección específicos para la supervigilancia del cumplimiento de la norma como la inspección de observadores (artículo 7 del Tratado) y las reuniones consultivas de los Estados firmantes para intercambiar informaciones, consultarse mutuamente sobre asuntos de interés común relacionados con la Antártica, y formular, considerar y recomendar a sus Gobiernos medidas para promover los principios y objetivos del presente Tratado, inclusive medidas relacionadas con el uso pacífico de la Antártica (artículo 9.1.a del Tratado). Se previene que los mecanismos específicos de protección sólo operan para los Estados parte del Tratado, los demás Estados si bien están sujetos al deber de cumplir con el uso pacífico de la Antártica como norma de ius cogens, sólo participan de los mecanismos de salvaguarda generales para este tipo de norma, es decir, aquellas obligaciones erga omnes que surgen consistentes en cesar la violación y sancionar a los transgresores. También a su respecto puede operar el mecanismo contemplado en el Capítulo VIII de la CONU, incluso si los Estados no son parte de la Organización, pues el artículo 2.6 de la Carta establece que La Organización hará que los Estados que no son Miembros de las Naciones Unidas se conduzcan de acuerdo con estos Principios en la medida que sea necesaria para mantener la paz y la seguridad internacionales.

Además el TA es evidencia de la existencia de una opinio iuris cogentis respecto de la prohibición del uso o amenaza de la fuerza, pues aunque el acuerdo ratificado o adherido sólo por algunos Estados de la comunidad internacional, en la redacción de su texto han participado plenipotenciarios reunidos en conferencia manifestando su consentimiento expreso en relación con el contenido de la regla consuetudinaria respectiva y las adaptaciones de ella al TA (Carter et al. 2003:128).

\subsection{La universalidad de las normas de ius cogens}

Esta característica se desprende a partir de la frase incluida en el artículo 53 de la CVDT, una norma imperativa de derecho internacional general es una norma aceptada y reconocida por la comunidad internacional de Estados en su conjunto. Aunque ya está presente su vocación de universalidad por pertenecer a la esfera del derecho internacional general (Virally 1966:13, Puceiro 2005:372, Betanzos 2009:113). Esta universalidad enfatiza que el ius cogens existe no para satisfacer las necesidades individuales de los Estados, sino el superior interés de la comunidad internacional en su conjunto.

El ius cogens es universalmente válido por fundarse en normas morales absolutas, dotadas de una validez objetiva, en todo tiempo y lugar, al margen del consentimiento que hayan prestado a su favor los Estados, individualmente considerados. La teoría tradicional acerca de la obligatoriedad de las normas del DI en virtud del consentimiento de los Estados no aplica en el caso de las normas perentorias de derecho internacional general, pues a su respecto no es necesario probar el consentimiento de los Estados; el consentimiento no es irrelevante, pero se presume (Kennedy 1987:71-72).

Se ha de distinguir entre creación o formación 
de la norma de ius cogens y la declaración o reconocimiento de su existencia: la aceptación y reconocimiento de la norma, tienen relación con un proceso declarativo de la existencia de la norma de ius cogens, más no con su creación. El que la norma de ius cogens sea aceptada y reconocida como tal por la comunidad internacional en su conjunto, significa que no se exige el voto unánime ni se acepta el veto minoritario. Si un Estado rehúsa aisladamente aceptar el carácter imperativo de una norma, incluso si es apoyado por algunos Estados, ello no puede afectar a la aceptación y reconocimiento del carácter imperativo de esa norma por la comunidad internacional en su conjunto. Tampoco la norma de ius cogens puede ser reconocida tan sólo por algunos sujetos de DI, actuando solos o en conjunto con otros, e imponer su interpretación a la mayoría del resto de los Estados. Todos los Estados están obligados por la norma de ius cogens, no obstante disientan de ella, pues la norma refleja los valores sobre los que están construidos la comunidad internacional. El ius cogens se funda en un profundo consenso moral que es meramente ilustrado por la aceptación de la comunidad internacional en su conjunto, por tanto un Estado puede ser obligado por la norma de ius cogens, incluso, sin o contra su consentimiento (Puceiro 2005:372 y 373).

Se ha de prevenir que la comunidad de Estados en su conjunto, no se refiere a la unanimidad de Estados del orbe, tampoco a una simple mayoría o un gran número de Estados, hay que considerar que la comunidad internacional abraza diferentes sistemas jurídicos construidos sobre diversas ideas morales. Por tanto, comunidad internacional de Estados en su conjunto, se refiere a la ética universal de la comunidad internacional existente tras ella, la cual denota el mínimo moral común de los Estados miembros de la comunidad internacional, dejando apartadas aquellas ideas o principios particulares que fundan $y$ representan a un determinado régimen sociopolítico. En otras palabras, muestra aquel consenso general existente sobre determinados valores esenciales del orden internacional y no la suma de las preferencias individuales de los Estados. La expresión comunidad de Estados en su conjunto, aunque imprecisa, evoca la idea de una especie de solidaridad y unidad profunda de la sociedad internacional que trasciende las

9 Fuera del análisis debieran quedar los fundamentalismos religiosos y los regímenes sociopolíticos basados en el oposiciones particulares que existan entre los Estados. No es una cuestión meramente numérica, sino que se ha de considerar también otros aspectos, tales como, la frecuencia, el volumen, la consistencia de las prácticas estatales, de las decisiones judiciales internas o internacionales, de los escritos de la doctrina, etc. (Alexidze 1981:245, 246 y 257, Puceiro 2005:372).

La humanidad aunque dividida en diferentes pueblos y naciones posee una unidad esencial, que son la base fundacional de la comunidad internacional y el DI contemporáneo. El principio que subyace en esta materia es el de unidad en la diversidad; existe una identidad esencial del ser humano, que es la misma en las diversas épocas y culturas de las distintas regiones del mundo, sin perjuicio de la propia evolución y de las particularidades de cada nación, v.g. son elementos esenciales de esta identidad la capacidad racional, la voluntad, la sensibilidad del ser humano; además, a lo largo de la historia el hombre ha tenido aspiraciones e ideales semejantes, v.g. la idea de democracia, de justicia, de protección de la dignidad humana, de paz ${ }^{9}$. En definitiva, la frase comunidad internacional en su conjunto se refiere a un conjunto de Estados, configurado por aquellos que sean representativos de los diferentes tipos de sociedades existentes en el mundo. La comunidad internacional de Estados en su conjunto es un ente nuevo con existencia y fines propios, sin perjuicio de los de sus componentes. Por encima de los sistemas sociales, ideológicos, económicos y jurídicos, el grado de desarrollo, organización e interdependencia de la comunidad internacional ha exteriorizado la existencia de intereses y valores vitales y comunes para la realización de ella como tal y de sus miembros, en un determinado histórico de su evolución. Por ello precisamente, la comunidad, a estos fines, es apreciada en su conjunto y no en su unanimidad absoluta (Alexidze 1981:245-247, 257, Puceiro 2005:367,372).

\section{PRUEBA DE LA OPINIO IURIS COGENTIS RESPECTO DEL USO PACÍFICO DE LA ANTÁRTICA}

La CIJ a propósito de las Actividades militares y paramilitares de Estados Unidos en Nicaragua (1986), para determinar el contenido

autoritarismo, pues coartan indudablemente, la voluntad, la libertad y la sensibilidad del ser humano. 
de la norma consuetudinaria de ius cogens que prohíbe el uso o la amenaza de la fuerza señala que existe una opinio juris respecto a la obligatoriedad de abstenerse del uso de la fuerza, la cual puede ser deducida de la actitud de las Partes y de los Estados respecto de ciertas resoluciones de la Asamblea General (vid. Tabla 1), y en especial de la Resolución 2625 (XXV) de 1970, Declaración relativa a los Principios de Derecho Internacional referentes a las Relaciones de Amistad y a la Cooperación entre los Estados de conformidad con la Carta de las Naciones Unidas. El consentimiento de las partes a esta resolución, en entendido de la Corte, no puede ser interpretado como una simple reiteración de los cometidos de la Carta, sino como una aceptación de las normas declaradas por la resolución; el principio del no uso de la fuerza, es un principio de derecho internacional consuetudinario, expresándose una opinio juris a su respecto, por lo que puede ser aplicada separadamente de las provisiones de la Carta, sobre todo al tratarse de un principio sustancial (CIJ 1986:188).

Para evidenciar la existencia de una opinio juris cogentis respecto de la norma que prohíbe el uso o amenaza de la fuerza en el territorio antártico, se han revisado Resoluciones de la Asamblea General de las Naciones Unidas atinentes. Se ha de prevenir que si bien se critica que el artículo 38 del Estatuto de la CIJ no hace alusión a este tipo de resoluciones como fuente del DI (Pellet 2006:711 y ss.), qué duda cabe de la importancia jurídico-política de las mismas, pues la Asamblea General es el máximo foro de discusión del sistema de Naciones Unidas y este hecho revela que lo discutido allí y los postulados que de allí emanan tienen un carácter a lo menos de consideración. Dado que la reiteración sobre el uso pacífico de la Antártica es tan frecuente en cantidad y en calidad que demuestra la existencia de una opinio iuris respecto de su carácter cogente.

$\mathrm{Si}$ bien han existido pronunciamientos al respecto desde la década de los cincuenta del siglo pasado, como el citado por parte de la India en 1956, a partir del $38^{\circ}$ período de sesiones, celebrado en 1983, se ha tratado el tema en 16 oportunidades, la última en 2005 , y en resoluciones ha sido reiterativa la afirmación del uso pacífico del territorio antártico (Villamizar 2012:285,286). Las expresiones que generalmente se utilizan en ese sentido son las siguientes:

Tabla 1. Listado de resoluciones en que se reconoce el uso pacífico de la Antártica y del tratado antártico.

Fuente: Villamizar, 2012:286.

\begin{tabular}{|c|c|c|c|c|}
\hline Sesión Asamblea General & Año & Resolución & $\begin{array}{c}\text { Afirmación uso pacífico de } \\
\text { la Antártica }\end{array}$ & $\begin{array}{c}\text { Alusión o reconocimiento del } \\
\text { Tratado Antártico }\end{array}$ \\
\hline 38 & 1983 & $38 / 77$ & $\mathrm{X}$ & \\
\hline 39 & 1984 & $39 / 152$ & $\mathrm{x}$ & $\mathrm{x}$ \\
\hline 40 & 1985 & $40 / 156$ & $\mathrm{x}$ & $\mathrm{x}$ \\
\hline 41 & 1986 & $41 / 88$ & $\mathrm{x}$ & $\mathrm{x}$ \\
\hline 42 & 1987 & $42 / 46$ & $\mathrm{x}$ & $\mathrm{x}$ \\
\hline 43 & 1988 & $43 / 86$ & $\mathrm{x}$ & $\mathrm{x}$ \\
\hline 44 & 1989 & $44 / 124$ & $\mathrm{x}$ & $\mathrm{x}$ \\
\hline 45 & 1990 & $45 / 78$ & $\mathrm{x}$ & $\mathrm{x}$ \\
\hline 46 & 1991 & $46 / 41$ & $\mathrm{X}$ & $\mathrm{x}$ \\
\hline 47 & 1992 & $47 / 57$ & $\mathrm{x}$ & $\mathrm{x}$ \\
\hline 48 & 1993 & $48 / 80$ & $\mathrm{x}$ & $\mathrm{x}$ \\
\hline 49 & 1994 & $49 / 80$ & $\mathrm{x}$ & $\mathrm{x}$ \\
\hline 51 & 1996 & $51 / 56$ & $\mathrm{x}$ & $\mathrm{X}$ \\
\hline 54 & 1999 & $54 / 45$ & $\mathrm{x}$ & $\mathrm{x}$ \\
\hline 57 & 2002 & $57 / 51$ & $\mathrm{x}$ & $\mathrm{x}$ \\
\hline 60 & 2005 & $60 / 47$ & $\mathrm{X}$ & $x$ \\
\hline
\end{tabular}


(i) Reafirmando su convicción de que, en interés de toda la humanidad, la Antártica debe seguir utilizándose exclusivamente con fines pacíficos y no ser teatro ni objeto de desacuerdos internacionales.

(ii) Reconociendo que el TA, en el que se estipula, entre otras cosas, la desmilitarización del continente, la prohibición de las explosiones nucleares y de la eliminación de desechos nucleares, la libertad de la investigación científica y el libre intercambio de datos científicos, promueve los propósitos $y$ principios de la Carta.

(iii) Teniendo presentes el TA y la importancia del sistema derivado de él.

Además, estos pronunciamientos sobre la Cuestión Antártica se han visto reforzados por otras resoluciones relativas al Hemisferio sur $y$ áreas adyacentes libres de armas nucleares ${ }^{10}$, en las cuales se destaca la importancia del TA como mecanismo que contribuye a liberar de armas nucleares el hemisferio sur y las áreas adyacentes.

Lo acontecido y pretendido en la Antártica en materia de su uso pacífico, es una tendencia que se refuerza en ciertos lugares físicos que tienen relevancia para la humanidad, tal y como ocurre con el espacio ultraterrestre, que si bien tiene un régimen jurídico diferente al antártico, la Asamblea General de las Naciones Unidas busca mediante sus Resoluciones ${ }^{11}$ que al igual que la Antártica, el espacio ultraterrestre se utilice de manera pacífica, pues la posibilidad del uso de la fuerza o amenaza de la misma llevaría en estas zonas llevaría a lo menos a carreras armamentistas desbocadas, que le restarían recursos a los Estados para suplir las necesidades fundamentales de su población.

\section{CONCLUSIONES}

1. El Tratado Antártico ha sido un mecanismo fundamental y eficaz para evitar confrontaciones bélicas entre los

10 n’s 62/35, 5 diciembre 2007; 63/49 y 63/65, 2 diciembre 2008; 63/44, 2 diciembre 2009; 63/58, 8 diciembre 2010.

11 ns 51/122, 13 diciembre 1996; 54/68, 6 diciembre
Estados que pretenden una parte o todo su territorio, y prueba de esto es la ausencia de conflicto en y por ese territorio desde le entrada en vigor del mismo. Al recordar que el TA se suscribió en una época muy tensa en las relaciones mundiales (en la cual los horrores de la Segunda Guerra estaban muy presentes, pero también en pleno escenario de la Guerra Fría, con todo lo que implicaba ese fenómeno), se debe reconocer que fue un avance muy significativo para la Humanidad y para el DI en materia de paz.

2. La prohibición del uso o amenaza de la fuerza es una norma de ius cogens. Evidencia de ello son las opiniones de la doctrina y jurisprudencia internacionales, en especial la de la CDI y la CIJ. La primera codificación del principio la encontramos tras las guerras mundiales, en el Pacto Briand-Kellogg de 1928 y en la CONU de 1945. El TA la codifica, especificando su contenido y creando mecanismos de protección especiales específicamente en su implementación en el ámbito del territorio antártico; además evidencia la existencia de una opinio iuris cogentis respecto del principio que prohíbe la amenaza o uso de la fuerza en salvaguarda de la paz y seguridad internacionales.

3. Las normas de ius cogens son imperativas, generales y universales, por tanto de aplicación obligatoria respecto de todos los Estados de la comunidad internacional. Aplicado este aserto al TA implica que la obligación de uso pacífico de la Antártica obliga a todos los Estados de la comunidad y no sólo a los 49 Estados que son parte del mismo.

3.1. Las normas de ius cogens son generales porque pertenecen al derecho internacional general o común, y como tales son oponibles a todos los Estados del orbe, pues salvaguardan intereses generales y comunes, como en caso de la prohibición de la amenaza o uso de la fuerza, la paz y 
seguridad internacionales.

3.2. La norma de ius cogens que prohíbe la amenaza o el uso de la fuerza como norma de origen consuetudinario se desdobla de sus reconocimientos expresos en tratados internacionales, y como fuente consuetudinaria existe y es autónoma a la fuente convencional, por tanto el deber de uso pacífico de la Antártica obliga también a quienes no son parte del TA o a los Estados que quitaran su consentimiento en obligarse a través del mecanismo de denuncia del Tratado.

3.3. La teoría tradicional acerca de la obligatoriedad de las normas del DI en virtud del consentimiento de los Estados no aplica en el caso de las normas perentorias de derecho internacional general, por tanto el consentimiento al uso pacífico de la Antártica se presume respecto de todos los Estados de la comunidad internacional, lo que significa que no se exige el voto unánime ni se acepta el veto minoritario. El ius cogens se funda en un profundo consenso moral que es meramente ilustrado por la aceptación de la comunidad internacional en su conjunto, por tanto un Estado puede ser obligado por la norma de ius cogens, incluso, sin o contra su consentimiento.

4. Las reiterativas alusiones al uso pacífico del territorio antártico concretadas en diversas resoluciones de la Asamblea General de las Naciones Unidas, son una muestra evidente de la formación de una opinio iuris cogentis, toda vez que la práctica general en el entendimiento de ese principio genera un carácter de obligatoriedad universal, y por lo tanto aunque Estado no sea parte del TA debe respetar los principios consagrados en él, fundamentalmente lo concerniente al uso pacífico del territorio antártico.

\section{AGRADECIMIENTOS}

Agradecemos a Ivonne Beltrán, quien nos ayudara a conseguir documentos del Departamento de Estado de Estados Unidos y de otras instituciones estatales de ese país.

\section{FUENTES DE CONSULTA}

a) Documentos

DEPARTMENT OF STATE. Memorandum (NOE), November 6 1947, Washington D. C.

DEPARTMENT OF STATE. Memorandum. Draft Communication Regarding the Antarctic. February 19, 1948a, Washington D. C.

DEPARTMENT OF STATE. Memorandum (NOE), February 25, 1948b, Washington D. C.

DEPARTMENT OF STATE. Memorandum (EUR), February 25, 1948c, Washington D. C.

DEPARTMENT OF STATE. Memorandum (EUR), March 24, 1948d, Washington D. C.

DEPARTMENT OF STATE. Memorandum (NOE), July 30, 1948e, Washington D. C.

DEPARTMENT OF STATE. Memorandum (EUR), August 3, 1948f, Washington D. C.

DEPARTMENT OF STATE, Bulletin, vol. 19, $\mathrm{n}^{\circ} 479$, September 5 1948g, Washington D. C. P. 301.

DEPARTMENT OF STATE. Note Verbale, Chilean Ministry of Foreign Affairs, October 7, 1948h, (attached to U.S. Santiago Embassy Dispatch No. 652, Octobre 11, 1948), Department of State file 800.014 (Antarctica/ 10-1148).

DEPARTMENT OF STATE, Policy Planning Staff, June 9, 1948i. Washington D. C.

DEPARTMENT OF STATE. Memorandum (EUR) AntarcticNext United States Steps. April 20, 1949, Washington D. C.

DEPARTMENT OF STATE. U. S. Draft Declaration on Antarctica. In Foreign Relations, 1950a, Vol. I, P. 905. Washington D.C.

DEPARTMENT OF STATE. Memorandum form the Embassy of the Soviet Union to the Department of State, June 8 1950b, in Foreign Relations 1950, Vol I. pp. 911 913. Washington D. C.

DEPARTMENT OF STATE. Memorandum "Modus Vivendi", March 5, 1951, in Foreign Relations, 1950. Vol. I. pp. 1717-1718. Washington D. C.

POLAR RECORD. (1948). Recent Argentine and Chilean Decrees Relating to the Antarctic [The texts of certain decrees relating to Argentine and Chilean territorial claims in the Antarctic were reproduced, with a map showing the extent of these claims, in the Polar Record, No. 82, July 1946, pp. 412-17]. The texts of some more recent decrees are printed below. It may be noted that the western limit of the Argentine claim, shown as 
longitude $68^{\circ} 34^{\prime} \mathrm{W}$. in the map. Volume 5, Issue 3536, December 1948, pp 223-227.

POLAR RECORD. (1949). Review of 'Territorial claims in the Antarctic' Polar Record, 5, pp 361-361 doi:10.1017/ S003224740004479X. No. 37-38, Jan-July 1949.

UNITED STATES NATIONAL ACADEMY OF SCIENCES. Antarctic Research Elements of a Coordinated Program. May 2 1949, Washington D. C. 24 pp.

b) Jurisprudencia

CIJ. Antarctica Case, United Kingdom v. Argentina, y United Kingdom $v$. Chile International Court of Justice (1956, Report of Judgment, Advisory Opinions and Orders (1956), Order of March 16th 1956.

CIJ. Case concerning military and paramilitary activities in and against Nicaragua (Nicaragua $v$. United States of America) (1986), sentencia, 27 junio 1986.

CIJ. Accordance with international law of the unilateral declaration of independence in respect of Kosovo (request for advisory opinion) (2010), opinión consultiva, 22 julio 2010.

c) Normas jurídicas

Carta de las Naciones Unidas (1945). La Ley $n^{\circ}$ 8.402, publicada el 3 de enero de 1946 Dispone cumplir y llevar a efecto como Ley de la República de Chile la Carta de las Naciones Unidas.

Convención de Viena sobre el Derecho de los Tratados. Viena, 23 de mayo de 1969. U.N. Doc A/CONF.39/27 (1969), 1155 U.N.T.S. 331, entrada en vigor 27 de enero de 1980. Promulgada en Chile por el Decreto Supremo del Ministerio de Relaciones Exteriores $\mathrm{n}^{\circ}$ 381, Diario Oficial, 22 de junio de 1981.

Pacto Briand-Kellogg o Pacto de París, París, 27 de agosto de 1928 .

Resolución $n^{\circ} 2625$ (XXV) de la Asamblea General de Naciones Unidas, de 24 de octubre de 1970, que contiene la Declaración relativa a los principios de Derecho internacional referentes a las relaciones de amistad y a la cooperación entre los Estados de conformidad con la Carta de las Naciones Unidas.

Resolución de la Asamblea General de Naciones Unidas 62/35, de 5 de diciembre de 2007.

Resolución de la Asamblea General de Naciones Unidas 63/44, de 2 de diciembre de 2009.

Resolución de la Asamblea General de Naciones Unidas 63/49, de 2 de diciembre de 2008.
Resolución de la Asamblea General de Naciones Unidas 63/58, de 8 de diciembre de 2010.

Resolución de la Asamblea General de Naciones Unidas 63/65, de 2 de diciembre de 2008.

Resolución de la Asamblea General de Naciones Unidas 51/122, de 13 de diciembre de 1996.

Resolución de la Asamblea General de Naciones Unidas 54/68 de 6 de diciembre de 1999.

Resolución de la Asamblea General de Naciones Unidas 59/2, de 20 de octubre de 2004.

Resolución de la Asamblea General de Naciones Unidas 61/101, de 17 de diciembre de 2007.

Resolución de la Asamblea General de Naciones Unidas 61/110 y 61/111, de 14 de diciembre de 2006.

Resolución de la Asamblea General de Naciones Unidas 62/217, de 22 de diciembre de 2007.

Resolución de la Asamblea General de Naciones Unidas 65/97, de 10 de diciembre de 2010.

Resolución de la Asamblea General de Naciones Unidas 65/271, de 7 de abril de 2011.

Resolución de la Asamblea General de Naciones Unidas 66/71 de 9 de diciembre de 2011.

Tratado Antártico. Suscrito en Washington, Estados Unidos, el $1^{\circ}$ de diciembre de 1959. Entró en vigencia el 23 de junio de 1961. Promulgado por Decreto Supremo N³61 del Ministerio de Relaciones Exteriores, Diario Oficial, 14 de julio de 1961 y rectificado en Diario Oficial, 2 de diciembre de 1961.

d) Impresos

Agüero, D. (2010). Roberto Guyer y su propuesta de creación de una entente antártica de los países del hemisferio sur. Estudios Hemisféricos y Polares, 1 (2), 90-105.

Alexidze, L. (1981). Legal nature of jus cogens in contemporary international law. Recueil des cours, 172 (3), 223-268.

Barros, M. (1990). Historia Diplomática de Chile (1541 -1938). (2 $2^{a}$ edición). Santiago: Editorial Andrés Bello.

Becker, F. (2010). Some Reflections on the Antarctic Treaty. Polar Record, 1, 2-4.

Berguño, J. (2009). Evolución y perspectivas del Sistema Antártico. ISTOR, Revista Internacional de Historia, 10 (39), 70-84.

Berguño, J. y Elzinga, A. (2010). The Achievements of the IGY. En Barr, S. y Lüdecke, C. (Comps.), From Pole to Pole: The History of the International Polar Years (IPYs). London: Springer,

Betanzos, E. (2009). Ius cogens. Revista USCS, Direito, 10 (17), 109-116. 
Blum, J. (1994). The deep freeze: Torts, Choice of Law, and the Antarctic Treaty Regime. Emory International Law Review, 8 (2), 667-699.

Boczek, A. (1984). The Soviet Union and the Antarctic Regime. American Journal of International Law, 78, 834858.

Carter, B., Trimble, P. y Bradley, C. (2003). International Law. (4a edición). New York: Aspen Publishers.

Dodds, K. (1997). Geopolitics in Antarctica: Views from the Southern Oceanic Rim. Chichester, UK: John Wiley.

Dodds, K. (2006). Post-colonial Antarctica: An Emerging Engagement. Polar Record, 1, 59-70.

Dodds, K. (2008). The Great Game in Antarctica: Britain and the 1959 Antarctic Treaty. Contemporary British History, 22 (1), 43-66.

Dodds, K. (2009). La administración del continente polar: los orígenes geopolíticos del Tratado Antártico de 1959. ISTOR, Revista Internacional de Historia, 10 (39), 27-49.

Gómez, A. (2003). El ius cogens internacional. Estudio histórico-crítico. México D.F.: Universidad Nacional Autónoma de México.

Haddelsey, S. (2012). Shackleton's Dream. Fuchs, Hillary and the Crossing of Antarctica. London: The History Press,

Hayton, R. (1956). The American Antarctic. American Journal of International Law, 50 (3), 590-592.

Hayton, R. (1960). The Antarctic Settlement of 1959. American Journal of International Law, 54 (2), 349371.

Hunter, W. (1951). The Antarctic Problem: an Historical and Political Study. London: George Allen and Unwin.

Jacobsson, M. (2011). Building the International Legal Framework for Antarctica En P. Berkman, M. Lang, D. Walton y O. Young (Eds.), Science Diplomacy. Antarctica, Science, and the Governance of International Spaces. Washington: Smithsonian Institution Scholarly Press.

Jiménez de Aréchaga, E. (1980). El Derecho Internacional Contemporáneo. Madrid: Editorial Tecnos.
Kennedy, D. (1987). The Sources of International Law. American University Journal of International Law and Policy, 2, 1-96.

Klots, F. (1990). America on ice. Antarctic Policy Issues. Washington: National Defense University Press.

Pellet, A. (2006). Article 38. En A. Zimmermann, C. Tomuschat, y K. Oellers-Frahm (Eds.) The Statute of the International Court of Justice. A Commentary. New York: Oxford University Press Inc.

Pinochet de la Barra, O. (1981). Evolución político-jurídica del problema antártico. Revista de Estudios Internacionales, 14 (55), 380-393.

Puceiro, R. (2005). Las normas de jus cogens en el campo del derecho internacional contemporáneo. En E. Jiménez de Aréchaga, H. Arbuet - Vignali, R. Puceiro (Eds.) Derecho Internacional Público. Principios, normas y estructuras (Vol. I) (pp. 359-385). Montevideo: Fundación de Cultura Universitaria.

Samuels, J. (2008). Condominium arrangements in international practice: reviving an abandoned concept of boundary dispute resolution. Michigan Journal of International Law, 29, 732-780.

Scott, K. (2003). Institutional developments within the Antarctic Treaty System. International \& Comparative Law Quartely. 52 (2), 473-487.

Scott, S. (2011). Ingenious and innocuous? Article IV of the Antartic Treaty as imperialism. Polar Journal, 1, 5162.

United Nations (1966). Yearbook of International Law Commission (Vol. I, part II). New York: United Nations.

Verdross, A. (1966). Jus dispositivum and jus cogens in International Law. American Journal of International Law, 60 (1), 55-63.

Villamizar, F. (2012). Tratado Antártico y Mecanismos de Protección del Territorio Antártico. International Law: Revista Colombiana de Derecho Internacional, 21, 255-295.

Virally, M. (1966). Réflexions sur le jus cogens. Annuaire Français de Droit International, 12, 5-29. 
